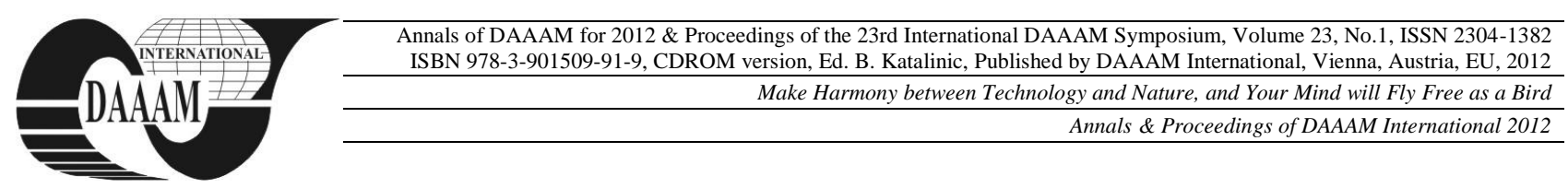

\title{
MONITORING SYSTEM FRAMEWORK AND ARCHITECTURE OVER SUPPLY CHAIN
}

\author{
ARUVAELI, T[anel]; SERG, R[isto]; KAARE, K[ati] \& OTTO, T[auno]
}

\begin{abstract}
Modern manufacturing develops towards sharing technological capabilities in the supply chain. Thus, it is necessary to monitor the manufacturing process concerning various facilities. Detection of machinery utilization, tracking machinable parts on pallets and pauses identification can provide additional information for production planning and increase manufacturing efficiency. Using wireless sensors and LabView programming environment, prototype for machinery status and pauses identification in supply chain was designed and introduced. RFID (Radio Frequency Identification) tags with sensors applicable to the part pallets or carriers were introduced for measuring continuous data of significant parameters throughout the supply chain.

Keywords: wireless sensor network, RFID, real time feedback, condition monitoring system, machinery utilization analysis, production planning, logistics in shop floor, data acquisition
\end{abstract}

\section{INTRODUCTION}

Effective production planning and scheduling are core competencies of competitive supply chains. Managing operations, whether in manufacturing or service sector, always require information about relevant properties of assets and other entities involved in operational process. Increasing complexity of industries has made reaching this aim highly challenging. Corporate consolidation, globalization and expanding product portfolios increase supply chain complexity while tightening competition, specific customer demands and difficult market economical situation obligate producers to meet challenging financial performance [1]. Accordingly, production planners and schedulers have to focus on several important goals: schedule the plant efficiently, keep inventories low, satisfy customer demand, and anticipate the impact of every decision on supply chain. They have to visualize the impact of planning decisions on the entire supply chain.

The basis of fluent work flow in the shop floor is raw material availability in working zone, clear working order and good state of machinery. The main component to achieve success in manufacturing enterprises is efficiency in work flow that creates the basis for high productivity. High productivity needs intelligent inputs as material flow logistics control, machinery state monitoring and machinery utilization monitoring for better planning. In a modern manufacturing system, flexibility is necessary. Key words of modern manufacturing systems are small batch size, flexibility, shortened production cycles, reduced work-in-progress, make-to-order and almost instantaneous delivery [2].
The problem in manufacturing is the lack of real time information about machining quality, production overview and logistics in shop floor and between subcontractors. Furthermore, the information cannot be shared in different databases for fast and functional usage. Thus, fast and informative feedback is needed from the shop floor to meet modern manufacturing system requirements. Need for technology based on real time wireless sensor network (WSN) monitoring system, employed by embedded computers, is becoming more critical than ever before. New supply chain monitoring technologies provide these possibilities and deliver necessary information to successfully optimize production in a complex and competitive marketplace. Increasing visibility between different supply chain members with better tracking clearly has potential to improve the effectiveness of all kinds of supply chains. The technology for collecting, distributing, and analyzing operational information has evolved during the past years. RFID is the new technology that is making inroads into this information collection process. Similar to a bar code, RFID is an automated identification and data collection technology that uses radio frequency waves to transfer data between readers and items with RFID devices. Compared to other ways of widely used tracking devices, RFID technology is more effective, and guarantees extensive tracking throughout the whole supply chain. Numerous supply chains - wide RFID tracking initiatives and applications in several industries [3] indicate the profitability of this technology, unfortunately applications in industries with minor role of retailers, such as the technical trade, do not exist.

The benefits of tracking include: real-time coordination of material flows and individually tracked items, such as merge-in-transit; providing an effective link between physical reality and information systems, such as improved inventory count and goods receipt transactions; and improved logistics management metrics and analyses. If RFID technology is used for tracking, it also enables the automation of operational supply chain processes and creates possibilities of providing information to support managerial processes. RFID based tracking systems are already being used in several industries from manufacturing to recycling and waste management.

Real time feedback from workbench helps to avoid unscheduled breaks, increases the lifetime of tools, provides feedback concerning work piece quality, saves environment and production time. Condition monitoring 
is a process of monitoring a system by studying certain selected parameters so that significant changes of those parameters are related to a developing failure [4]. Condition monitoring is one of the major components of predictive maintenance. The use of condition monitoring enables scheduling maintenance or other actions to be taken to avoid the consequences of failure before the failure occurs [5].

Many techniques have been employed and different sensors used for monitoring the condition of machineries. In-process surface roughness model was developed based on cutting forces measured with dynamometer in turning [6] and in ball-end milling [7]. Vibrations and acoustic have been used as tools for detecting working modes in turning [8]. Also drilling has been studied for condition monitoring application [9]. Infrared thermography measurements have been investigated as good indicator in CNC milling. Machinery utilization has been detected with high reliability based on acoustic and vibration information in laser cutting and in CNC milling [10].

Smart Machine program has been introduced as manufacturing control system that helps in process planning, tool condition monitoring and health and maintenance [11]. It is essential to collect data from all workbenches in one database for cognitive and simple use. Modern machines often have preinstalled databases for utilization information, but keeping the information in separate machines does not provide a clear overview and productivity does not rise considerably.

The aim of the research is to collect all the monitoring information wirelessly into one database, automatically analyze it, present the information in real time UI and also in historical view for further analysis. This paper mainly investigates the wireless data acquisition techniques and monitoring architecture up to collecting data in a database, as well as presenting data. Data analysis is not covered in the current paper.

This paper covers three monitoring applications: machinery condition monitoring; machinery utilization monitoring and pause identification; and material and products flow tracking.

The aim of the current paper is to introduce the developed production monitoring system framework and architecture. Methods of condition monitoring, machinery utilization monitoring and material logistics control are explained by means of practical work.

\section{SUPPLY CHAIN MONITORING FOR MACHINERY AND METAL ENGINEERING SECTOR}

RFID technology facilitates major innovation to supply chain management. RFID have been identified as potential cost reduction technology and used to track product flow. RFID is used to identify inbound and outbound products, as well as to locate products in warehouses, to track and locate work in progress and completed products in manufacturing and processing industries, to monitor and track products in transit, to guarantee replenishment of store shelves and to help in reverse logistics.

The barcode is the most frequently used technology in tracking, although the use of technically advanced but more expensive RFID is gradually increasing. One of the main advantages of using RFID technology instead of barcodes is that no line of sight is needed in reading the tagged items. There are no typical barcode environment challenges like dirt and wear while RFID tracking is used. Radio waves can read the data even through lorry walls. One substantive benefit of RFID technology compared to the barcode technology is that all RF identifiers are unique. There are three essential parts in an RFID tracking system: the tag (or "transponder"), the reader device, and the back-end computer system. In many applications, RFID tags have to be placed on metallic surfaces, but typical label-type tags with dipolelike antennas do not work efficiently on metallic surfaces.

While using RFID technology in solving critical problems, most passive RFID tags cannot be placed on metal objects as metallic surface changes its radiation pattern, input impedance, radiation efficiency and resonant frequency. Through detailed theoretical analysis, relationship between the distance to the metallic surface and the tag's communication efficiency is deduced.

Several independent researchers have verified a classical problem: the performance of passive UHF (Ultra High Frequency) RFID tags degrade when placed near a metal surface. This is an unfortunate situation, since industry continues to seek for a low-cost way to tag metal assets. Microstrip-based antennas modified for operation with RFID offer a useful alternative, but are considerably more expensive to fabricate. Often, industry simply provides a thin (e.g., 3 to $6 \mathrm{~mm}$ ) low dielectric material such as flexible foam in order to provide enough separation from the metal surface to provide adequate, though severely degraded performance. A foam separation of $3.2 \mathrm{~mm}$ can yield a performance degradation of $16 \mathrm{~dB}$ or more over free-space performance, resulting in reduction in read distance of about $85 \%$ [12].

A passive UHF RFID system consists of a transponder, also called a tag, and an interrogator, also called a reader. The reader provides power via RF energy, commands via protocol, and timing. The tag consists of an IC (integrated circuit) and an antenna. The tag communicates by modulating the IC impedance, which changes the scattering characteristics of the antenna, which can be detected by the reader. For an RFID tag to operate, the IC must receive sufficient power to run the circuitry and provide enough backscatter signal strength for the reader to detect the response. Regarding several systems it can be assumed that the system is limited in the forward channel (reader to tag), and if the tag responds, then the reader will detect the response. As is common with many antennas, the bandwidth of an RFID tag is typically limited by the impedance of the antenna. The reactive IC impedance can further aggravate this problem. Commercial RFIDs use a printed 
(or "ribbon," or "flat") dipole because of the low manufacturing cost involved. Furthermore, they are commonly limited to about $92 \mathrm{~mm}$ in length in order to fit comfortably on a $101.6 \mathrm{~mm}$ wide label. At $915 \mathrm{MHz}$, a half-wave resonant dipole is approximately $160 \mathrm{~mm}$ long, therefore the antenna is electrically short and narrow. Thus, the antenna has a large quality factor $\mathrm{Q}$ (nominally 15) and is relatively sensitive to the environment.

In the current research wireless monitoring network is combined with the latest RFID technology, and can be used in machining industry. The patent pending solution of Confidex Ironside ${ }^{\mathrm{TM}}$ on-metal Gen2 UHF tag provides a trusted benchmark for low-cost passive RFID in its size and durability to various customer requirements. Ironside's performance enables several on-metal identification challenges to be solved, which were earlier impossible with passive RFID. The tag can be mounted easily on any metallic surfaces either mechanically with screws or pop rivets, or with industrial adhesive. For a superior grip, Confidex also offers a welding bracket for tags, which can be used in spot welding the tag onto the surface. As well as being able to survive various challenging environments from being dropped under water to tropical weather and mechanical impacts, Ironside has passed the rigorous testing requirements for the Aerospace Standard AS5678 specification as the first tag in the market. The tag is successfully used in tracking assets from steel pallets to train wagons. Ironside is the most square shaped of all the tags tested and as a result, performed well on metal with the tag in different orientations using the far field antenna. However, when affixed to the plastic-encased removable hard drive, the read distance dropped from over 24 feet down to below 6 feet, while other medium range tags maintained a much more consistent performance. With the near field antenna, Ironside was the last in all tests.

Given the performance on plastic, Ironside is not recommended for tracking reusable plastic containers. However, on-metal the tag performs well in small spaces. Ironside's strengths can be set forth on-metal in extreme temperatures where a lot of memory on the tag is required.

\section{MONITORING ARCHITECTURE}

Machinery monitoring is beneficial for both: machineries and human competence. Efficiency in production depends on productivity of workstations. Productivity is concerned as the effective and efficient utilization of resources (inputs) in producing goods (output) [13]. Preconditions for productivity are technological capabilities and human competences. Machinery monitoring can develop both preconditions (Fig. 1). It helps to maintain machines in an optimized schedule, avoid inappropriate machining modes and change tools in time. Additionally, it provides information for more efficient production and human resources planning.

Monitoring is part of a modern performance improvement cycle (Fig. 2). It means cooperation

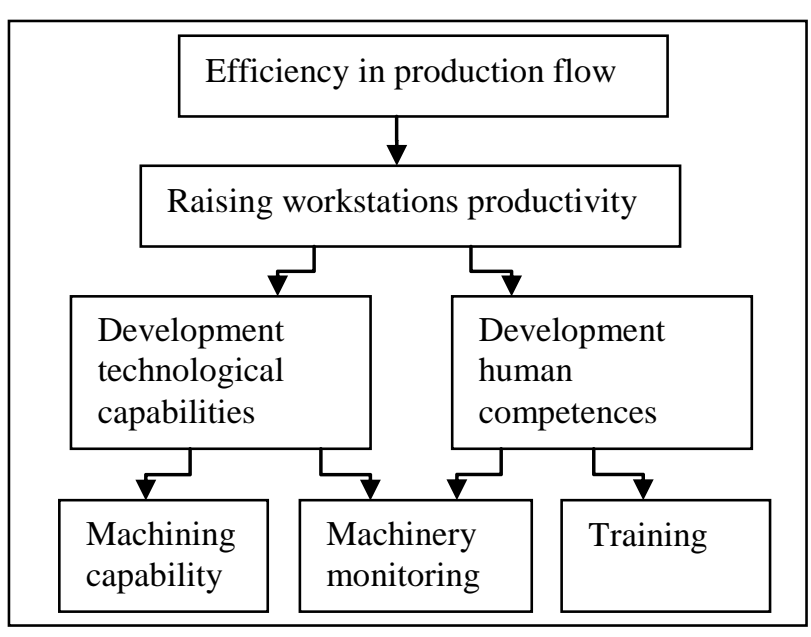

Fig. 1. Efficiency tree in production flow

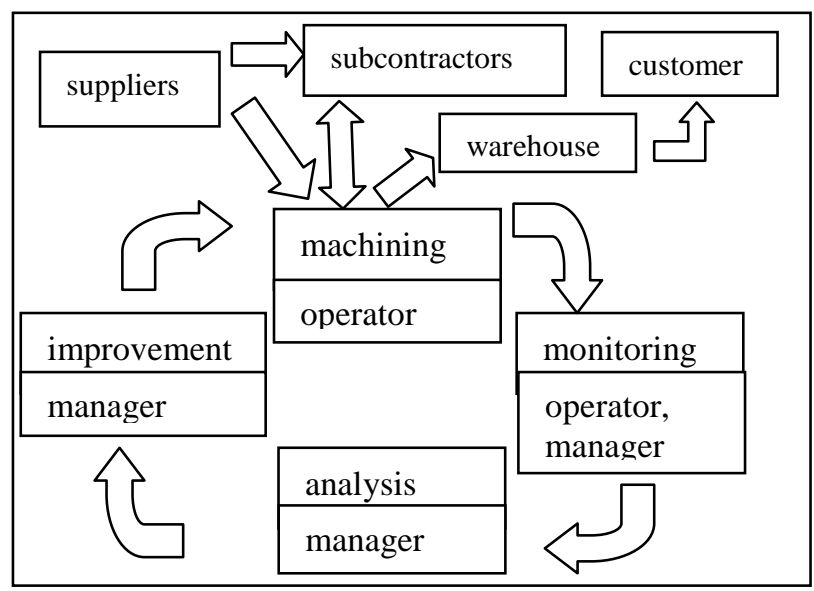

Fig. 2. Monitoring cycle

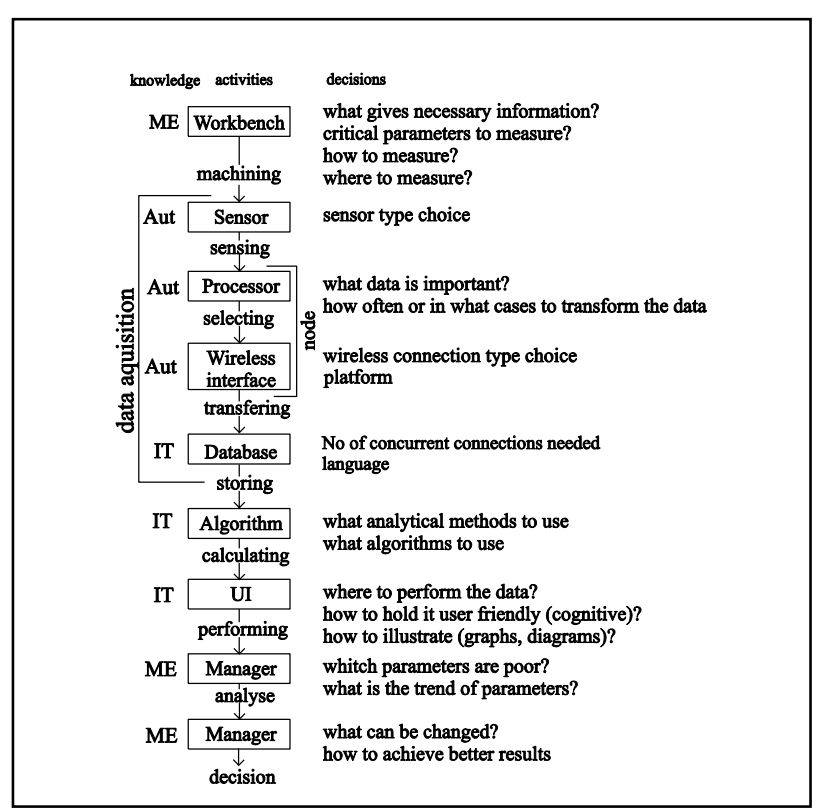

Fig. 3. Main activities and interdisciplinary knowledge needed in monitoring structure.

between an operator and a manager. The manager analyzes results and the operator comments and makes improvement decisions suggested by the operator. Monitoring system set up is a complicated process with multiple activities that require complicated engineering 
knowledge. It requires interdisciplinary cooperation between mechanical engineers (ME), automation engineers (Aut) and information technology engineers (IT). For each activity many decisions have to be made to develop an energy efficient, user friendly and accurate system (Fig. 3).

The data acquisition system consists of the following components: a measurement node with sensor, a wireless gateway and a database for storing measurement results.

The measurement node performs actual measurement at predefined sampling rate and sends it to the gateway. The gateway saves the received data in the database in real time. Process visualization and situation analysis is elaborated in a separate system based on data stored in the database.

Scalability and ease of use were important system design aspects. Often used simple file based data storage was excluded due to poor interoperability and concurrent use write access difficulties. In the current architecture a readily available RDBMS (Relational Database Management System) PostgreSQL was used for data storage. This database system can be accessed via standard SQL (Structured Query Language) statements, as it easily handles tens of concurrent connections and solves data storage, replication, and backup challenges. Data is arranged as tables consisting of records (rows) and fields (columns). One RDBMS instance can handle several databases in case of data separation needs, different access rights (login roles) for different users and enough tables for data organizing.

Usage of standard SQL database as data abstraction layer enables users to define their own data presentation or connection layer that suits their exact needs. Several interfacing solutions are freely available. The possibilities of interfacing include but are not limited to display real time machinery status and update of real time production state or supply chain state to ERP (Enterprise Resource Planning) system. As all data collected from sensors is saved in the database it is easy to conduct historical or fault analysis and update production models. Situation analysis is simplified by this solution as data from different sources is saved in a single storage and can be fused easily.

The measurement system can consist of heterogeneous mix of nodes. In the current study, National Instruments (NI) WSN nodes together with LabView software were used. Custom LabView program was designed to interface measurement node to SQL database via TCP/IP network. A similar solution can be used for other types of measurement nodes. The measurement system gateway and database system may run on the same computer in different concurrent processes. The system may also be distributed where the database server is located in a different site and accessed via standard TCP/IP network.

\section{PRACTICAL WORK}

The utilization monitoring and pause identification prototype model was designed and preparations were made for implementing it in shop floor for lathe. The spindle bearing temperature information was collected as one of lathe condition monitoring indicators.

\subsection{Utilization monitoring and pause identification}

Wireless shop floor machinery utilization monitoring and pause identification sample model was designed and programmed for presenting machinery utilization and pause identification information in real time. Sample monitoring model kit includes 3 lathe models each equipped with micro motor and NI node WSN-3202. They were wirelessly linked to NI gateway WSN-9791 and processor with UI screen. Lathe model body includes WSN node, micro motor, batteries for micro motor, switch for lathe control, relay and wiring between components. NI graphical programming environment LabWiev was used to create the program for monitoring, to design UI (Fig. 4; Fig. 5) for presenting utilization information in real time.

Additionally 3 OFF-(ON) (normally open, closed only if held down) buttons and one LED (Light Emitting Diode) were attached to one model for pause identification. Buttons were labelled "Planned maintenance", Fault" and "No order". The appliance automatically switches on the LED in case the machine does not work (spindle is not turning), but is switched on from the main switch. The LED should turn on if normal jig or work piece set up time has been exceeded. It means, in about 5 minutes after the spindle has stopped. LED informs the operator about malfunction in production. The operator presses a button for the purpose of analysis until LED switches off. The button has to be held up to 2 seconds as nodes receive information once in a second and feedback from the gateway is sent back once in a second, as well. "Planned maintenance" means that machinery maintenance is planned beforehand and it does not interrupt the production plan. The button "Fault" signifies fault in machinery or unplanned maintenance that interrupts production plans. The button "No order" indicates that the operator lacks work order, drawing or raw material.

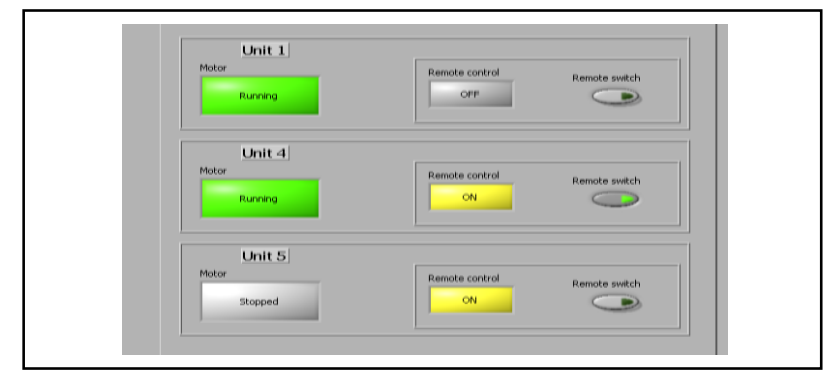

Fig. 4. UI, main view: workbenches utilization information

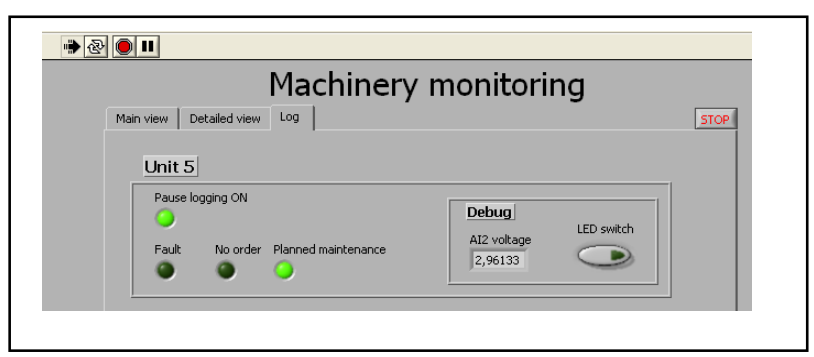

Fig. 5. UI, Investigation of pauses 


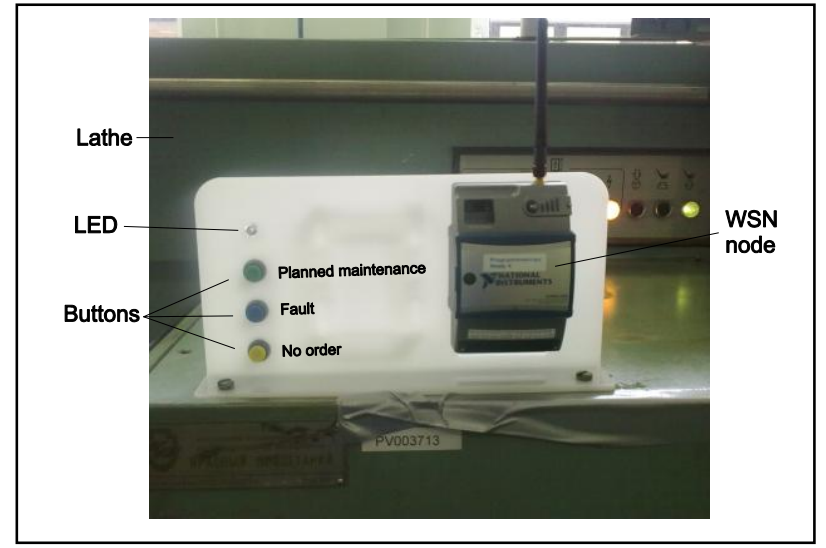

Fig. 6. Pause identification feedback panel for operator

For utilization monitoring and pause identification implementation in shop floor, acrylic feedback panel with buttons, LED and NI node WSN-3202 was designed (Fig. 6). Thus, spindle rotation speed information as input for utilization detection and LED as indicator for operator can be applied. For that purpose Hall effect sensor was attached between gearbox and spindle to read spindle rotation speed. Hall effect sensor was linked to Defendec node.

\subsection{Lathe bearing temperature monitoring}

The lathe temperature was measured in 2 points with J-type thermocouples. NI WSN-3212 4ch 24bit Thermocouple Input Node was used for real measurements. The first thermocouple was placed in contact with spindle front bearing housing from inside the gearbox; the second thermocouple was placed in contact with spindle front bearing flanged housing between gearbox and spindle. The data acquisition interval was $1 \mathrm{~s}$ and all the results were saved in real time in SQL database table with the following field layout:

1. Timestamp - holding time of measurement

2. Sequence - measurement sequence No in the current test

3. NodeID - ID of the measurement node (for saving measuring results of different nodes to one table)

4. Value1 - actual measured value1 (currently the temperature of the first thermocouple)

5. Value2 - actual measured value2 (currently the temperature of the second thermocouple)

In total, 9 experiments were conducted, 3 in each speed. The spindle was set free running at speeds 160 , 600 and $1200 \mathrm{rpm}$ for $300 \mathrm{~s}$ and then left to standstill for 300 s. Sample set from measured data values are in table 1. The measurement results of the temperature dynamics at different spindle speeds can be viewed in Fig 7.

\begin{tabular}{|c|c|c|c|}
\hline sequence & node & value1 & value2 \\
\hline 0 & 2 & 29,048 & 30,107 \\
\hline 1 & 2 & 29,032 & 30,103 \\
\hline 2 & 2 & 29,015 & 30,098 \\
\hline 3 & 2 & 28,615 & 30,014 \\
\hline 4 & 2 & 28,492 & 29,988 \\
\hline 5 & 2 & 28,299 & 29,981 \\
\hline
\end{tabular}

Tab. 1. Sample set of measurement values from database

As can be expected, the spindle rotation heats up the bearing. The temperature of the gearbox side of the cylindrical roller bearing is lower as it is cooled by the circulating transmission oil. Effects of heating and cooling of the bearing can be seen most easily at the highest test speed $(1200 \mathrm{rpm})$ where the temperature difference during the test reached $10^{\circ} \mathrm{C}$. The cooling effect at standby mode can be easily observed, as the difference between bearing and ambient temperature is more significant. The sudden temperature decrease at the startup of the spindle and temperature increase at the stop of the spindle can be explained by start and stop of the cooling oil circulation.

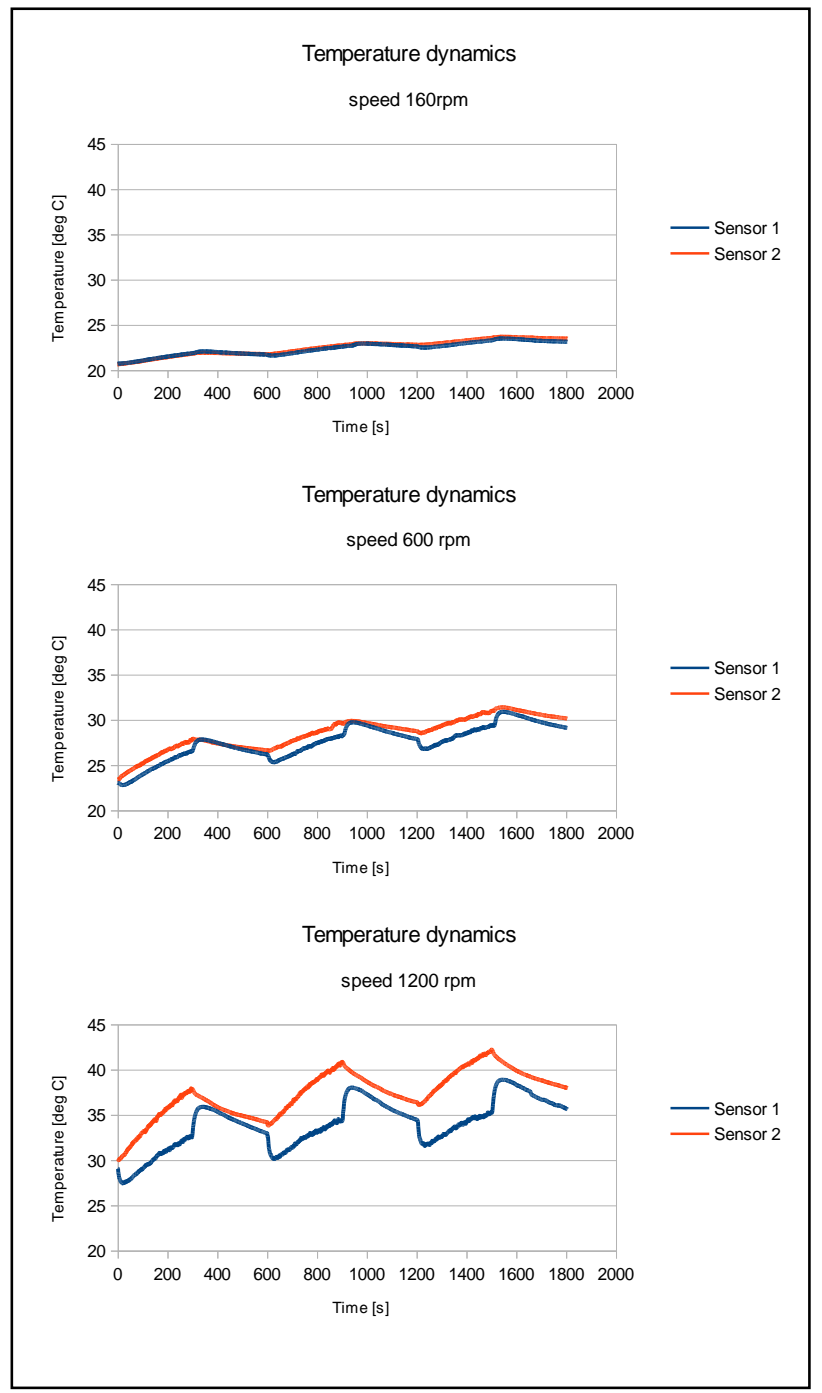

Fig. 7. Temperature dynamics at different spindle speeds

It can be noted that even by this very limited measured dataset some situation detection of the turning can be made. The positive temperature gradient at magnitude of $1 \mathrm{deg} / \mathrm{min}$ indicates the rotation of the spindle and the negative temperature gradient indicates the idleness of the spindle.

\section{DISCUSSION}

Defendec node and NI nodes were used in the monitoring application. NI hardware and software are easier to use than the Defendec node, as NI equipment is preinstalled and the programming environment is graphical. The advantage of using Defendec nodes is lower cost and wider opportunities in programming. Additionally, Defendec nodes permit to read high 
frequency measurements, more than 2000 samples/s. NI nodes allow reading one input per second. This excludes NI nodes usage measuring fast changing information as acoustic, vibrations and rotational speed. Nevertheless, it is sufficient for temperature, voltage and current monitoring. In advanced intelligent WSN systems and in high frequency measurements Defendec nodes are more suitable with their flexibility, on the other hand the usage of NI nodes is often more efficient in research.

Based on the developed prototype model, utilization monitoring and pause identification application can be implemented on workbenches in shop floor. The pause identification application efficiency depends on operator, currently. The LED signals the operator to press a button, but the LED can be ignored. A stronger pause identification version would eliminate the possibility to continue machining without pressing one of the buttons. Still, the filter free automatic feedback about pause roots is the aim.

Recent researches for machinery condition monitoring and its relations with work piece quality have provided us a lot of methods for monitoring. The system to make monitoring in shop floor easy to follow and cognitive has not been established yet. Missing is the system that covers entire supply chain or cluster [14]. It should monitor all the machineries, including their maintenance data and also logistics data of materials, semi finished parts and products in real time. Finally, the collected data needs to be presented in cognitive and simple to follow user interface.

The described measurement system architecture makes it possible to add other measurement and sensing nodes. More reliable and complex situation detection can be achieved by adding ambient temperature and electrical supply current measurement information to the temperature dataset. Adding user feedback data about idle times to the same database adds even more information that can be used for increasing production efficiency.

The aim of the research is to implement the ultimate wireless monitoring system that increases efficiency in manufacturing enterprises. The next step is to implement the prototype solution in the shop floor and compare the productivity before and after monitoring implementation. For this solution, analysis of different condition monitoring data has to be studied to create recognizable patterns for a trustworthy machinery condition feedback.

\section{CONCLUSION}

A wireless machinery utilization monitoring and pause identification prototype model was designed and recommendations to implement it in shop floor were made. The machinery maintenance monitoring architecture was analyzed and its structure was implemented in the shop floor to complement it with more sensors. Proposals for logistics monitoring using RFIDs were given to track materials and products movement between subcontractors and suppliers.

Practical basis was designed for ultimate real time wireless monitoring system for manufacturing companies. It covers all workbenches with utilization, pause identification and their condition information.
Additionally, (semi)products tracking is proposed as part of the monitoring system

\section{ACKNOWLEDGEMENTS}

This research was supported by the Estonian Ministry of Education, Research Project SF0140113Bs08 and the Estonian Science Foundation (Grant F7852).

\section{REFERENCES}

[1] Kõrbe, K.; Kuhi, K. \& Koppel, O. (2012). Measuring temperature and water content in road structures with sensor equipped RFID tags, Proceedings of the 8th International Conference of DAAAM Baltic Industrial Engineering, 8th International Conference of DAAAM Baltic Industrial Engineering, Tallinn, Estonia, ISBN 978-9949-23-265-9, Otto, T. (Ed.), pp. 315 - 320, DAAAM Baltic, Tallinn

[2] Viswanadham, N. \& Srinivasa Raghavan N. R. (1997). Flexibility in Manufacturing enterprises, Sãdhanã, Vol. 22, 1997, pp. 135163

[3] Hinkka, V. \& Tätilä, J. (2011), Implementing RFID tracking in the technical trade supply chain, 23rd Annual Conference of the Nordic Logistics Research Network, Harstad, Norway

[4] Marwala, T. (2012). Condition Monitoring Using Computational Intelligence Methods, Springer, ISBN 978-1-4471-2379-8, London

[5] Aruväli, T.; Serg, R.; Preden, J. \& Otto T. (2011), In-process determining of the working mode in CNC turning, Est. J. of Eng., Vol. 17, 2011, pp. 4-16

[6] Tangjitsitcharoen, S. \& Senjuntichai, A. (2010), Monitoring of surface roughness in CNC turning process, Annals of DAAAM for 2010 \& Proceedings, 21st International DAAAM Symposium, Zadar, Croatia, ISSN 1726-9679, Katalinic, B. (Ed.), pp. 13911392, DAAAM International, Vienna, Austria

[7] Tangjitsitcharoen, S. \& Senjuntichai, A. (2010), In-process monitoring and prediction of surface roughness in ball-end milling process, Annals of DAAAM for 2010 \& Proceedings, $21 \mathrm{st}$ International DAAAM Symposium, Zadar, Croatia, ISSN 17269679, Katalinic, B. (Ed.), pp. 1389-1390, DAAAM International, Vienna, Austria

[8] Aruväli, T; Otto, T. \& Preden, J. (2010), Modern monitoring opportunities in shop floor, Annals of DAAAM for 2010 \& Proceedings, 21st International DAAAM Symposium, Zadar, Croatia, ISSN 1726-9679, Katalinic, B. (Ed.), pp. 989-990, DAAAM International, Vienna, Austria

[9] Shin, B. \& others (2006), A Web-based machining process monitoring system for E-manufacturing implementation. $J$. Zhejiang Univ. SCIENCE A, Vol. 7, 2006, pp.1467-1473

[10] Astapov, S.; Preden, J. S.; Aruväli, T \& Gordon, B. (2012), Production machinery utilization monitoring based on acoustic and vibration signal, Proceedings of the 8th International Conference of DAAAM Baltic Industrial Engineering, 8th International Conference of DAAAM Baltic Industrial Engineering, Tallinn, Estonia, ISBN 978-9949-23-265-9, Otto, T. (Ed.), pp. 268 - 273, DAAAM Baltic, Tallinn

[11] Atluru, S.; Huang, S. H. \& Snyder, J. P. (2012), A smart machine supervisory system framework. Int. J. Adv. Manuf. Technol., Vol. 58, 2012, pp. 563-572

[12] Mohammed, N. A.; Sivakuvar, M. \& Deavours, D. D. (2009), An RFID tag capable of free-space and on-metal operation, Proceedings of radio \& wireless symposium, Radio \& wireless symposium, San Diego, CA, ISBN 978-1-4244-2698-0, pp. 6366, San Diego, CA

[13] Riives, J.; Otto, T. \& Lõun, K. (2007) Methods for enhancing productivity and work efficiency in the workshop. J. of Machine Eng., Vol. 7, 2007, pp. 86-95

[14] Lõun, K.; Otto, T. \& Riives, J. (2009). E-manufacturing concept solution for tooling sector. Est. J. of Eng., Vol. 15, No 2, 2009, pp. $108-120$ 